\title{
Changes in serum liver enzymes level after switching from stavudine/lamivudine to zidovudine/lamivudine in NNRTIs based anti-retroviral regimens in Hawassa, Southern Ethiopia
}

\author{
Demissie Assegu Fenta, Agete Tadewos Hirigo* \\ Hawassa University College of Medicine and Health Science Teaching Hospital, Hawassa, Southern Ethiopia
}

Email addresses:

demissieasegu@yahoo.com (D. Assegu), agetetadewos@yahoo.com (A. Tadewos)

To cite this article:

Demissie Assegu Fenta, Agete Tadewos Hirigo. Changes in Serum Liver Enzymes Level after Switching from Stavudine/Lamivudine to Zidovudine/Lamivudine in NNRTIs Based Anti-Retroviral Regimens in Hawassa, Southern Ethiopia. American Journal of Health Research. Vol. 2, No. 6, 2014, pp. 397-403. doi: 10.11648/j.ajhr.20140206.23

\begin{abstract}
Background: During stavudine phase-out in a resource limited countries, Zidovudine or Tenofovir is used to substitute stavudine. However, data concerning any difference in liver enzymes level after therapy change (switching) in Ethiopia is very limited. Methods: This prospective cohort study was carried out from May 2013 to July 2014 at ART clinic of Hawassa University teaching hospital. Of hundred fifty HIV-infected; immunologically stable adults receiving triple antiretroviral therapy: 120 were patients receiving stavudine based regimen with either of efavirenz or nevirapine during ART initiation (switch group); and the rest 30 patients were receiving zidovudine based regimen with either of efavirenz or nevirapine and also never switched (control group). Lamivudine is common for both groups. Levels of serum liver enzymes were determined and hepatotoxicity assessed according to World Health Organization ART guideline. Statistical analysis was done using Statistical Package for Social Sciences (SPSS) Version 20. Results: Serum mean AST and ALT level in the switch group decreased significantly over the time observed ( $\mathrm{p}=<0.0001$ for both enzymes); however ALP level was significantly increased $(\mathrm{p}=<0.0001)$. Hepatotoxicity in the switch group was significantly decreased (AST grade $\geq 1$ from $35.8 \%$ to $15.8 \%$; and ALT from $20.8 \%$ to $3.3 \%$ ) after 12 month of therapy change. Conclusion: Significantly a decreased hepatotoxicity was observed at the 12 month of post switch and this indicated that a substitution of stavudine to Zidovudine could have a potential to reverse hepatotoxicity. However, significantly decreased white blood cells count and abnormally increased level of ALP enzyme should require periodic monitoring and further investigations.
\end{abstract}

Keywords: Hepatotoxicity, HAART Switching, HIV/AIDS, Hawassa, Ethiopia

\section{Introduction}

The introduction of highly active antiretroviral therapy (HAART) has led to a marked reduction in acquired immunodeficiency syndrome (AIDS) related morbidity and mortality [1]. Since its introduction patients have started to live longer however, co-morbid problems are challenging human immunodeficiency virus (HIV) infected patients. Among these, hepatotoxicity is the one which is associated with antiretroviral drugs and it may be life threatening [2]. Antiretroviral drugs-related liver injury is a common cause of morbidity, it may result treatment discontinuation in HIVinfected patients [1,3]. Elevations in serum hepatic enzymes: aspartate aminotransferase (AST), alanine aminotransferase
(ALT) and alkaline phosphatase (ALP) have been described in association with all major classes of HAART [4]. However, the complexity of medication used in antiretroviral therapy (ART) complicates the understanding of the independent effects of each drug in the development of drug induced liver injury [4, 5]. All antiretroviral classes are associated with hepatotoxicity, though this is more commonly seen with the non-nucleoside reverse transcriptase inhibitors (NNRTIs) [6, 7]. Nevirapine (NVP) increases the risk of hepatotoxicity and monitoring hepatic enzymes is recommended if feasible, especially for women with HIV who have CD4 cell counts $>250$ cells $/ \mathrm{mm} 3$ and individuals with HIV who are co-infected with hepatitis-B virus (HBV) or hepatitis-C virus (HCV) [8]. However, hepatotoxicity due to hepatic steatosis 
resulting from mitochondrial toxicity: more common with stavudine (d4T) than with other nucleoside reverse transcriptase inhibitors (NRTIs) [9].

The aim of the present study was to determine changes in serum liver enzymes level as markers of hepatotoxicity in HIV-infected patients those who switching from d4T-based regimen to zidovudine (AZT) antiretroviral (ARV) drugs in a resource limited setting.

\section{Methods}

\subsection{Study Design}

This prospective cohort study was carried out from May 2013 to July 2014 at ART clinic of Hawassa University teaching hospital.

\subsection{Study Setting and Study Population}

Eligible patients were HIV-infected, immunologically stable adults receiving only triple antiretroviral therapy that included d4T backbone with one of NNRTI (either EFV or NVP) during HAART initiation. Cases were only patients who came for switching from d4T to AZT based regimen; whereas control were patients using AZT based regimen with either of EFV or NVP during HAART initiation and those never switched before were included. Both groups were similar in duration of HAART exposure and age range. All participants included were $\geq 18$ years of age, receiving HAART for a minimum of one year and have a good ART adherence (adherence rate $\geq$ $95 \%$ ). A good adherence is defined by missing $<2$ dose of 30 doses or $<3$ dose of 60 doses; and it was adopted from Ethiopian Federal Ministry of Health (EFMOH), HIV Care/ART follow-up form. Participants receiving antiTuberculosis drugs, pregnant women, and jaundiced patients, patients with a known liver disease, $\mathrm{HBV}$ and $\mathrm{HCV}$ positives, and renal failures were excluded.

\subsection{Data Collection and Measurements}

For all participants, data were collected on the socio- demographic information together with body weight and height. Blood sample was collected from each participant at the time of HAART switch and at 12 month of the switch by using Plain tubes and $\mathrm{K}_{2}$ EDTA anticoagulated tubes. $\mathrm{CD}^{+}$ lymphocyte count was done by using flow cytometry instrument (Becton Dickinson, CA, USA), and also CELLDYN 1800 was used to perform haematological parameters. Clotted blood was centrifuged at 3000 cycles/ minute, and then serum was obtained for liver enzymes, total protein (TP) and albumin (ALB) tests. Serum AST, ALT and ALP were assayed by enzymatic method; and serum total TP and ALB through colorimetric method, (Human Gesellschaft für Biochemica und Diagnostica mbH, Germany). Hepatitis B virus (HBV) was done by two site sandwich immunoassay to determine surface antigen ( $\mathrm{HBsAg}$ ) from serum and for Hepatitis $\mathrm{C}$ virus (HCV) rapid immuno-chromatographic test was done using direct binding test for the visual detection of $\mathrm{HCV}$ antibodies in the serum. Severity grading of selected parameters of laboratory toxicities were assessed according to World Health Organization (WHO 2007) ART guideline, as follows:-

\subsubsection{Liver Enzymes (AST, ALT and ALP)}

Toxicity of degree-0: the level of toxicity which is considered as normal in which its value is $\leq 1.25 \times \mathrm{ULN}$ (upper limit normal) value. Toxicity of degree-1: the level of toxicity which is considered as weak in which its value is $1.25-2.5 \times$ ULN value. Toxicity of degree-2: the level of toxicity which is considered as moderate in which its value is 2.5- 5 x ULN value. Toxicity of degree-3: the level of toxicity which is considered as severe in which its value is 5 $-10 \times$ ULN value. Toxicity of degree-4: the level of toxicity which is considered as severe in which its value is $>10 \mathrm{x}$ ULN value.

\subsubsection{Haematological Parameters}

The below table indicates the toxicity grading of haematological parameters (hemoglobin concentration and platelets count) for HIV-infected patients who were on ART.

Haematological parameters toxicities

\begin{tabular}{|c|c|c|c|c|}
\hline & Grade-1 & Grade-2 & Grade-3 & Grade-4 \\
\hline $\begin{array}{l}\text { Haemoglobin } \\
\text { Platelets }\end{array}$ & $\begin{array}{c}8.0-9.4 \mathrm{~g} / \mathrm{dl} \\
(75-99) \times 10^{3} / \mathrm{mm}^{3}\end{array}$ & $\begin{array}{c}7.0 .-7.9 \mathrm{~g} / \mathrm{dl} \\
(50-74.999) \times 10^{3} / \mathrm{mm}^{3}\end{array}$ & $\begin{array}{c}6.6-6.9 \mathrm{~g} / \mathrm{dl} \\
(20-49.999) \times 10^{3} / \mathrm{mm}^{3}\end{array}$ & $\begin{array}{c}<6.6 \mathrm{~g} / \mathrm{dl} \\
<20 \times 10^{3} / \mathrm{mm}^{3}\end{array}$ \\
\hline
\end{tabular}

\subsubsection{Outcome Assessment}

The mean percentage changes of AST, ALT, ALP and other parameters at the switch time and at 12 month of the switch for each individual was assessed by using Van Leth and colleagues' formula [10]. For each patient at specific study period we calculated this estimate as follows:

$\%$ Increase of each parameter $=$ $\frac{- \text { Concentration at month }(\mathrm{X})-\text { Concentration at month }(\mathrm{A})}{\text { Concentration at month }(\mathrm{A}) a} \times 100$

Where, month-X is the study end point at which follow up takes place (12 month of HAART switch) and; month-A is the time of HAART switch or switch zero month.

\subsection{Statistical Analysis}

Data entry and Database management was completed using EPI-INFO 2002. Statistical analyses were done using Statistical Package for Social Sciences (SPSS) Version 20. Both chi-square test and fisher exact test were used for categorical variables. Mean values and standard deviations were tabulated for normally distributed variables; median values and interquartile range (IQR) were tabulated for 
skewed variables. Comparison of quantitative variables at the switch baseline and after 12 month of AZT replacement was performed with paired student $t$-test or Mann-Whitney $U$-test for those variables did not follow normal distribution. The alpha level was set at 0.05 for significance.

\subsection{Ethical Considerations}

The study was approved by the institutional review board (IRB) of Hawassa University College of Medicine and Health Science, Written informed consent was obtained from all participants.

\section{Results}

\subsection{General Characteristics of Study Population}

Of the 157 (100 females and 57 males) patients were enrolled in the study; 2 patients were lost to follow-up due to transferred out. Four patients were HBV positives and 1 was HCV positive. Therefore data of these 7 patients were not included in this analysis.

Table 1. Characteristics of the 150 study population

\begin{tabular}{|c|c|}
\hline Characteristic & Participant $(n=150)$ \\
\hline \multicolumn{2}{|l|}{ Sex: } \\
\hline Female, n $(\%)$ & $100(66.7)$ \\
\hline Male, n (\%) & $50(33.3)$ \\
\hline Age (years), mean $\pm \mathrm{SD}$ & $39.2(8.7)$ \\
\hline $20-30, \mathrm{n}(\%)$ & $24(16.0)$ \\
\hline $31-40, \mathrm{n}(\%)$ & $59(39.3)$ \\
\hline $41-50, \mathrm{n}(\%)$ & $51(34.0)$ \\
\hline $51-60, \mathrm{n}(\%)$ & $12(8.0)$ \\
\hline$>60, \mathrm{n}(\%)$ & $4(2.7)$ \\
\hline Weight $(\mathrm{Kg})$, mean $\pm \mathrm{SD}$ (min-max) & $57.9 \pm 11.6(38-91)$ \\
\hline BMI $\left(\mathrm{Kg} / \mathrm{m}^{2}\right)$, mean $\pm \mathrm{SD}(\min -\max )$ & $21.7 \pm 3.3(14.4-36.4)$ \\
\hline Below $18 \mathrm{Kg} / \mathrm{m}^{2}$, n (\%) & $12(8.0)$ \\
\hline $18-24.9 \mathrm{Kg} / \mathrm{m}^{2}, \mathrm{n}(\%)$ & $110(73.3)$ \\
\hline$\geq 25 \mathrm{Kg} / \mathrm{m}^{2}, \mathrm{n}(\%)$ & $18(18.7)$ \\
\hline HAART (months), mean \pm SD (min-max) & $60.8 \pm 13.4(17-91)$ \\
\hline$<24$ months, $\mathrm{n}(\%)$ & $1(0.7)$ \\
\hline 24-48 months, n (\%) & $32(21.7)$ \\
\hline 49-72 months, n (\%) & $89(59.3)$ \\
\hline$\geq 73$ months, $\mathrm{n}(\%)$ & $28(18.7)$ \\
\hline $\mathrm{Hb}(\mathrm{g} \%)$, mean $\pm \mathrm{SD}$ & $14.0(1.9)$ \\
\hline PLT count $\left(\right.$ cells $\left./ \mathrm{mm}^{3}\right)$, mean $\pm \mathrm{SD}$ & $2787(84.5)$ \\
\hline
\end{tabular}

\begin{tabular}{ll}
\hline Characteristic & Participant $(\mathbf{n}=150)$ \\
\hline WBC count $\left(\right.$ cells $\left./ \mathrm{mm}^{3}\right)$, mean \pm SD & $6.3(1.9)$ \\
CD4+ count $\left(\right.$ cells $\left./ \mathrm{mm}^{3}\right)$, median $(\mathrm{IQR})$ & $548(411-785)$ \\
CD4+ count $<350, \mathrm{n}(\%)$ & $26(17.3)$ \\
CD4+ count $35-500, \mathrm{n}(\%)$ & $37(24.7)$ \\
CD4 $>500, \mathrm{n}(\%)$ & $87(58.0)$ \\
AST, mean \pm SD & $38.1(8.8)$ \\
ALT, median $(\mathrm{IQR})$ & $33(24-44)$ \\
ALP, mean \pm SD & $216.7(88)$ \\
TP, mean $\pm \mathrm{SD}$ & $9.5(1.3)$ \\
ALB, mean \pm SD & $4.49(0.46)$ \\
\hline
\end{tabular}

N.B: BMI, body mass index; HAART, highly active antiretroviral therapy; $\mathrm{Hb}$, hemoglobin; PLT, platelets; WBC, white blood cells; CD4+, clusters of differentiation- 4 positive cells; SD, standard deviation AST, Aspartate aminotransferase; ALT, alanine aminotransferase; ALP, Alkaline phosphatase; TP, Total protein; ALB, Albumin

Base line characteristics of 150 HIV-infected patients (females $66.7 \%$ and males $33.3 \%$ ) had a mean age of 39.2 years. From 120 of the cases (switch-group), 44 (33.7\%) were on $\mathrm{d} 4 \mathrm{~T} / 3 \mathrm{TC} / \mathrm{EFV}$, and $76(63.3 \%)$ were on $\mathrm{d} 4 \mathrm{~T} / 3 \mathrm{TC} / \mathrm{NVP}$ regimens before switching to AZT/3TC based regimens. Of 30 patients of control group, 13(43.3\%) were on AZT/3TC/EFV and 17(56.7\%) were on AZT/3TC/NVP. At the time of the AZT/3TC replacement, the mean BMI was $21.7 \mathrm{Kg} / \mathrm{m}^{2}$ (range, 14.4-36.4 Kg/m²), and the BMI was remained stable throughout the 12 months following up without showing a significant change..Majority of patients $(81.3 \%)$ had BMI $<25 \mathrm{Kg} / \mathrm{m}^{2}$ (Table 1 ).

\subsection{Hepatotoxicity and Trends of Serum Liver Enzymes and Other Parameters (Switch Group vs. Control Group)}

At the switch 0 month serum levels of mean AST and ALT were significantly higher in the switch group when compared to control group $(\mathrm{P}=0.04$ and $\mathrm{P}=0.02)$, respectively. However ALP and TP were significantly higher in control group when compared to the switch group $(\mathrm{p}=<0.0001$ and $\mathrm{p}=0.005)$, respectively. On the other hand at the end of the study follow-up, the mean AST was significantly higher in the control group, $\mathrm{p}=0.01$ (table2).

Table 2. Comparison of Serum liver enzymes and other parameters of study population.

\begin{tabular}{|c|c|c|c|c|c|c|}
\hline \multirow{2}{*}{ Parameters } & \multicolumn{2}{|c|}{ At switch baseline (0-month) } & \multirow[b]{2}{*}{ p-value } & \multicolumn{2}{|c|}{ At the 12 month of post-switch } & \multirow[b]{2}{*}{ p-value } \\
\hline & Switch group & Control group & & Switch group & Control group & \\
\hline AST (U/L), mean \pm SD & $39.5(19.2)$ & $32.2(12.6)$ & 0.04 & $29.3(16)$ & $36.3(16.7)$ & 0.01 \\
\hline AST $\geq$ grade- 1 toxicity, $\mathrm{n}(\%)$ & $43(35.8)$ & $5(16.7)$ & 0.03 & $19(15.8)$ & $7(23.3)$ & 0.23 \\
\hline $\mathrm{ALT}(\mathrm{U} / \mathrm{L})$, mean $\pm \mathrm{SD}$ & $40(26.6)$ & $29(16.7)$ & 0.02 & $27.4(14.7)$ & $35.6(21)$ & 0.09 \\
\hline ALT $\geq$ grade-1 toxicity, n (\%) & $25(20.8)$ & $4(13.3)$ & 0.25 & $4(3.3)$ & $4(16.7)$ & 0.01 \\
\hline $\operatorname{ALP}(\mathrm{U} / \mathrm{L})$, mean $\pm \mathrm{SD}$ & $201(73.8)$ & $280(112)$ & $<0.0001$ & $245.5(83)$ & $257(98)$ & 0.47 \\
\hline ALP $\geq$ grade-1 toxicity, $\mathrm{n}(\%)$ & $2(1.6 \%)$ & $5(16.7)$ & 0.04 & $9(7.5)$ & $3(10.0)$ & 0.70 \\
\hline$(\mathrm{AST}+\mathrm{ALT})$ grade-1 toxicity, n (\%) & $17(14.1)$ & $4(13.3)$ & 0.96 & $3(2.5)$ & $2(6.7)$ & 0.59 \\
\hline $\mathrm{Hb}(\mathrm{g} \%)$, mean $\pm \mathrm{SD}$ & $14.5(1.7)$ & $12.2(1.7)$ & $<0.0001$ & $14.6(1.9)$ & $13(1.5)$ & $<0.0001$ \\
\hline PLT count $\left(\right.$ cells $\left./ \mathrm{mm}^{3}\right)$, mean $\pm \mathrm{SD}$ & $279(81.5)$ & $269(97)$ & 0.94 & 279(97) & $260(61)$ & 0.52 \\
\hline $\mathrm{WBC}$ count $\left(\right.$ cells $\left./ \mathrm{mm}^{3}\right)$ mean $\pm \mathrm{SD}$ & $6.4(1.9)$ & $6.1(2.1)$ & 0.38 & $5.9(1.9)$ & $6.1(1.6)$ & 0.71 \\
\hline $\mathrm{T} / \mathrm{P}(\mathrm{g} / \mathrm{dl})$, mean $\pm \mathrm{SD}$ & $9.3(1.3)$ & $9.9(1.5)$ & 0.005 & $10.1(0.8)$ & $9.9(1)$ & 0.52 \\
\hline $\mathrm{ALB}(\mathrm{g} / \mathrm{dl})$, mean $\pm \mathrm{SD}$ & $4.5(0.4)$ & $4.5(0.7)$ & 0.10 & $4.9(0.32)$ & $4.6(0.72)$ & 0.006 \\
\hline weight $(\mathrm{Kg})$, mean $\pm \mathrm{SD}$ & $57.4(11)$ & $60.1(13)$ & 0.30 & $56.9(11.5)$ & $56.4(11)$ & 0.78 \\
\hline BMI $\left(\mathrm{Kg} / \mathrm{m}^{2}\right)$, mean $\pm \mathrm{SD}$ & $21.6(3.4)$ & $22(3.2)$ & 0.36 & $21.5(3.4)$ & $21.8(3.4)$ & 0.76 \\
\hline
\end{tabular}




\begin{tabular}{|c|c|c|c|c|c|c|}
\hline \multirow{2}{*}{ Parameters } & \multicolumn{2}{|c|}{ At switch baseline (0-month) } & \multirow[b]{2}{*}{ p-value } & \multicolumn{2}{|c|}{ At the 12 month of post-switch } & \multirow[b]{2}{*}{ p-value } \\
\hline & Switch group & Control group & & Switch group & Control group & \\
\hline CD 4 count $\left(\right.$ cells $\left./ \mathrm{mm}^{3}\right)$, mean \pm SD & $613(268)$ & $485(243)$ & 0.01 & $593(290)$ & $532(222)$ & 0.41 \\
\hline HAART duration (months) mean $\pm \mathrm{SD}$ & $60.6(13.6)$ & $62(13.6)$ & 0.76 & - & - & - \\
\hline Age in years, mean $\pm \mathrm{SD}$ & $38.6(8.5)$ & $41.9(9.2)$ & 0.87 & - & - & - \\
\hline
\end{tabular}

N.B: BMI, body mass index; HAART, highly active antiretroviral therapy; Hb, hemoglobin; PLT, platelets; WBC, white blood cells; CD4+, clusters of differentiation-4 positive cells; AST, Aspartate aminotransferase; ALT, alanine aminotransferase; ALP, Alkaline phosphatase; TP, Total protein; ALB, Albumin.

\subsection{Within a Group Comparison of Hepatotoxicity and \\ Trends of Serum Liver Enzymes and Other Parameters}

The serum mean AST and ALT level in the switch group decreased significantly over the time observed $(\mathrm{p}=<0.0001$ at 12 month for both enzymes); however the mean ALP level was significantly increased ( $\mathrm{p}=<0.0001)$. WBC count in the switch group was significantly decreased $(\mathrm{p}=0.002)$. On the other hand the mean CD4+ T lymphocyte count in the control group significantly increased $(\mathrm{p}=0.001)$. Moreover TP and ALB mean value showed significant change in the switch group ( $\mathrm{p}=<0.0001$ ). Hepatotoxicity of AST and ALT enzymes of grade- 1 and above in the switch group was significantly decreased (AST from 35.8\% to $15.8 \%$ and ALT from $20.8 \%$ to $3.3 \%$ ). conversely ALP toxicity was significantly raised from $1.6 \%$ to $7.5 \%$ (table-3 and figure 1 ).

Table 3. Within a group comparison of Serum liver enzymes and other parameters of study population

\begin{tabular}{|c|c|c|c|c|c|c|}
\hline \multirow{2}{*}{ Parameters } & \multicolumn{2}{|l|}{ Switch-group } & \multirow{2}{*}{ p-value } & \multicolumn{2}{|c|}{ Control-group } & \multirow{2}{*}{ p-value } \\
\hline & Switch (0 month) & Switch 12-month & & 0 month & 12-month & \\
\hline $\mathrm{AST}(\mathrm{U} / \mathrm{L})$, mean $\pm \mathrm{SD}$ & $39.5(19.2)$ & $29.3(16)$ & $<0.0001$ & $32.2(12.6)$ & $36.3(16.7)$ & 0.27 \\
\hline AST $\geq$ grade- 1 toxicity, $\mathrm{n}(\%)$ & $43(35.8 \%)$ & $19(15.8)$ & & $5(16.7)$ & $7(23.3)$ & \\
\hline $\operatorname{ALT}(\mathrm{U} / \mathrm{L})$, mean $\pm \mathrm{SD}$ & $40(26.6)$ & $27.4(14.7)$ & $<0.0001$ & $29(16.7)$ & $35.6(21)$ & 0.28 \\
\hline ALT $\geq$ grade- 1 toxicity, $\mathrm{n}(\%)$ & $25(20.8)$ & $4(3.3)$ & & $4(13.3)$ & $4(16.7)$ & \\
\hline $\mathrm{ALP}(\mathrm{U} / \mathrm{L})$, mean $\pm \mathrm{SD}$ & 201(73.8) & $245.5(83)$ & $<0.0001$ & $280(112)$ & 257(98) & 0.41 \\
\hline$(\mathrm{AST}+\mathrm{ALT})$ grade-1 toxicity, $\mathrm{n}(\%)$ & $17(14.1)$ & $3(2.5)$ & & $4(13.3)$ & $2(6.7)$ & \\
\hline $\mathrm{Hb}(\mathrm{g} \%)$, mean $\pm \mathrm{SD}$ & $14.5(1.7)$ & $14.6(1.9)$ & 0.71 & $12.2(1.7)$ & $13(1.5)$ & 0.02 \\
\hline $\mathrm{Hb} \geq$ grade- 1 toxicity & $0(0.0)$ & $2(1.6 \%)$ & & $2(6.6)$ & $1(3.3)$ & \\
\hline PLT count $\left(\right.$ cells $\left./ \mathrm{mm}^{3}\right)$, mean \pm SD & $279(81.5)$ & 279(97) & 0.96 & 269(97) & $260(61)$ & 0.70 \\
\hline PLT $\geq$ grade- 1 toxicity & $1(0.8 \%)$ & $3(2.5 \%)$ & & $1(3.3)$ & $1(3.3)$ & \\
\hline WBC count $\left(\right.$ cells $\left./ \mathrm{mm}^{3}\right)$ mean \pm SD & $6.4(1.9)$ & $5.9(1.9)$ & 0.002 & $6.1(2.1)$ & $6.1(1.6)$ & 0.95 \\
\hline $\mathrm{T} / \mathrm{P}(\mathrm{g} / \mathrm{dl})$, mean $\pm \mathrm{SD}$ & $9.3(1.3)$ & $10.1(0.8)$ & $<0.0001$ & $9.9(1.5)$ & $9.9(1)$ & 0.9 \\
\hline weight $(\mathrm{Kg})$, mean $\pm \mathrm{SD}$ & $57.4(11)$ & $56.9(11.5)$ & 0.14 & $60.1(13)$ & $56.4(11)$ & 0.29 \\
\hline BMI $\left(\mathrm{Kg} / \mathrm{m}^{2}\right)$, mean $\pm \mathrm{SD}$ & $21.6(3.4)$ & $21.5(3.4)$ & 0.79 & $22(3.2)$ & $21.8(3.4)$ & 0.74 \\
\hline CD4 count $\left(\right.$ cells $\left./ \mathrm{mm}^{3}\right)$, mean $\pm \mathrm{SD}$ & $613(268)$ & $593(290)$ & 0.29 & $485(243)$ & $532(222)$ & 0.001 \\
\hline
\end{tabular}

N.B: BMI, body mass index; HAART, highly active antiretroviral therapy; Hb, hemoglobin; PLT, platelets; WBC, white blood cells; CD4+, clusters of differentiation-4 positive cells; AST, Aspartate aminotransferase; ALT, alanine aminotransferase; ALP, Alkaline phosphatase; TP, Total protein; ALB, Albumin; Kg, kilogram.

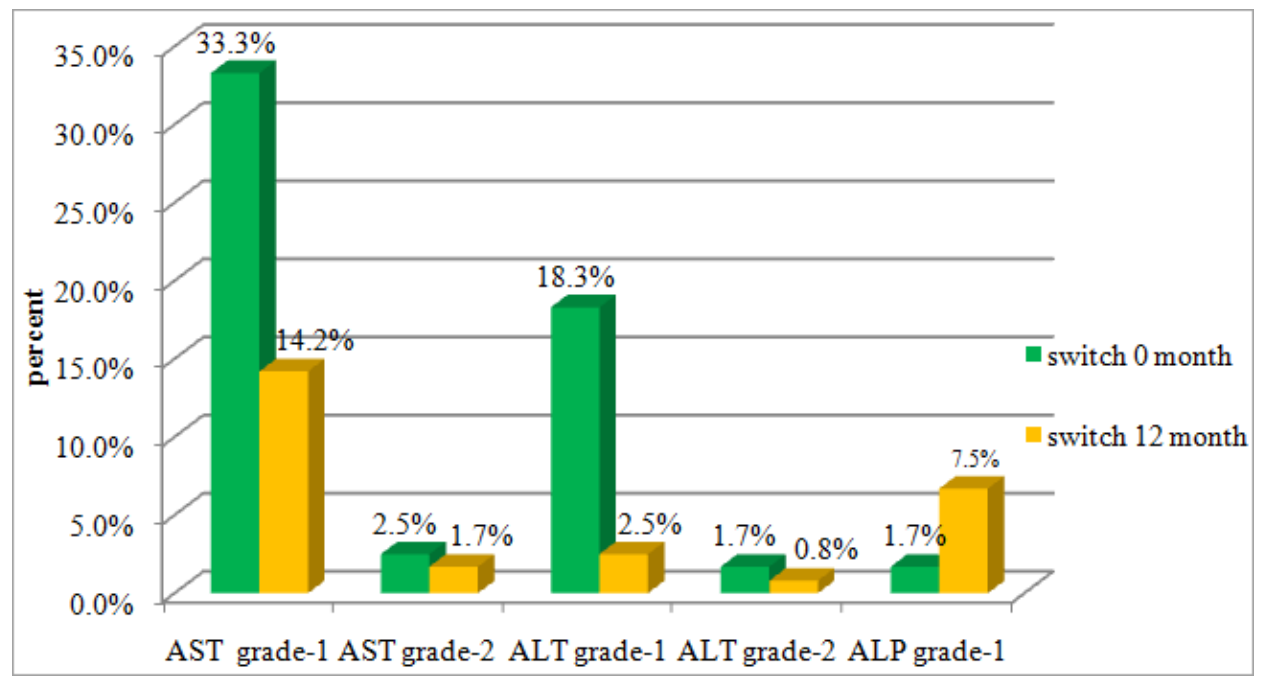

Figure 1. Hepatotoxicity indicator serum liver enzymes grade of the switch group. 


\subsection{Comparison of Change of Parameters with Different Variables in the Switch-Group}

Males have significantly higher mean AST and ALT value at the switch 0 month when compared with females, (46.9 vs. 36.3 \pm 11.0 ; $\mathrm{p}=0.005$ and 52.9 Vs. 34.3; $\mathrm{p}=<0.0001$ ) respectively. Mean ALP was significant with age $\geq 40$ years; however ALT was significant with patients using NVP (table 4).
Based on WHO hepatotoxicity grading, AST grade 1 in EFV group was $31.8 \%$ at the time of HAART switch and it was decreased to $13.6 \%$ after 12 month of post switch. However this AST grade 1 in NVP group also decreased from $34.2 \%$ to $14.6 \%$. In contrast to AST and ALT, ALP grade- 1 toxicity showed increasing trend at 12 month of post switch in EFV and NVP groups (figure 2).

Table 4. Change of parameters in some independent variables after 12 months

\begin{tabular}{|c|c|c|c|c|c|c|c|c|c|c|}
\hline Variables & * mean & AST \% (SE) & *mean & $\begin{array}{l}\text { ALT \% } \\
(\mathrm{SE})\end{array}$ & *mean & $\begin{array}{l}\text { ALP } \\
\%(\mathrm{SE}) \\
\end{array}$ & * mean & $\begin{array}{l}\text { TP \% } \\
\text { (SE) } \\
\end{array}$ & * mean & $\begin{array}{l}\text { ALB \% } \\
\text { (SE) }\end{array}$ \\
\hline Gender $=$ female & 36.3 & $-23.8(3.9)$ & 34.3 & $-18.1(4.0)$ & 204.5 & $32.8(5.8)$ & 9.4 & $9.1(1.7)$ & 4.5 & $8.4(1.5)$ \\
\hline male & 46.9 & $-9.1(13)$ & 52.9 & $-18.5(8.9)$ & 192 & $24.3(4.1)$ & 9.2 & $10.3(1.7)$ & 4.5 & $8.2(1.3)$ \\
\hline$p$ value & 0.005 & 0.20 & $<0.0001$ & 0.95 & 0.41 & 0.35 & 0.32 & 0.66 & 0.24 & 0.94 \\
\hline $\mathrm{BMI}=<25 \mathrm{Kg} / \mathrm{m}^{2}$ & 40.2 & $-22(3.7)$ & 40.2 & $-19.8(4)$ & 197 & $31.4(4.8)$ & 9.4 & $9.3(1.4)$ & 4.5 & $8.1(1.2)$ \\
\hline$\geq 25 \mathrm{Kg} / \mathrm{m}^{2}$ & 36.7 & $2.1(22)$ & 39.3 & $-10(10.4)$ & 218 & $23.4(7.9)$ & 9.2 & $10.5(3.7)$ & 4.4 & $9.6(1.9)$ \\
\hline$p$ value & 0.45 & 0.12 & 0.89 & 0.35 & 0.22 & 0.53 & 0.46 & 0.71 & 0.36 & 0.62 \\
\hline CD4+cells $\geq 500$ cells $/ \mathrm{mm}^{3}$ & 38 & $-14.5(7.1)$ & 38.7 & $-13.7(5.4)$ & 200 & $32.8(5.9)$ & 9.5 & $7.7(1.7)$ & 4.5 & $8.3(0.8)$ \\
\hline$p$ value & 0.28 & 0.29 & 0.50 & 0.14 & 0.98 & 0.42 & 0.18 & 0.074 & 0.29 & 0.96 \\
\hline $\mathrm{Hb}<12 \mathrm{~g} \%$ & 30.7 & $-6.7(18.2)$ & 26.7 & $21.9(40.2)$ & 186 & $32.8(19.7)$ & 9.5 & $9.8(3.5)$ & 4.4 & $13.6(5)$ \\
\hline $\mathrm{Hb} \geq 12 \mathrm{~g} \%$ & 39.8 & $-19(5)$ & 40.5 & $-19(3.8)$ & 201 & $30.1(4.3)$ & 9.3 & $9.5(1.3)$ & 4.5 & $8.1(1.1)$ \\
\hline$p$ value & 0.35 & 0.65 & 0.31 & 0.06 & 0.68 & 0.91 & 0.80 & 0.96 & 0.49 & 0.39 \\
\hline PLT $<150 \times 10^{3}$ cells $/ \mathrm{mm}^{3}$ & 37.2 & $22.3(24.2)$ & 44.2 & $-1.9(21.2)$ & 184 & 29.7(30.9) & 9.0 & $18.9(9.2)$ & 4.3 & $-9.2(28)$ \\
\hline PLT $\geq 150 \times 10^{3}$ cells $/ \mathrm{mm}^{3}$ & 39.6 & $-20(4.9)$ & 39.9 & $-18.7(3.9)$ & 201 & $30.2(4.2)$ & 9.4 & $9.1(1.3)$ & 4.5 & $8.9(0.75)$ \\
\hline $\mathrm{WBC} \geq 4 \times 10^{3}$ cells $/ \mathrm{mm}^{3}$ & 39.7 & $-16.8(5.4)$ & 41 & $-20.3(3.7)$ & 202.8 & $29.5(4.3)$ & 9.3 & $9.8(4.5)$ & 4.5 & $8.2(1.2)$ \\
\hline$p$ value & 0.82 & 0.34 & 0.28 & 0.14 & 0.42 & 0.68 & 0.58 & 0.48 & 0.22 & 0.71 \\
\hline NNRTI $=$ EFV based & 36.8 & $26.6(4.5)$ & 33.7 & $-20.1(4.1)$ & 188.6 & $28.6(4.9)$ & 9.4 & 7.1(1.9) & 4.4 & $8.6(1.0)$ \\
\hline NVP based & 41.2 & $-13.9(6.9)$ & 43.7 & $-17.0(5.7)$ & 207 & $31.1(6.0)$ & 9.3 & $10.9(1.7)$ & 4.5 & $8.2(1.6)$ \\
\hline$p$ value & 0.22 & 0.22 & 0.04 & 0.71 & 0.17 & 0.78 & 0.95 & 0.16 & 0.07 & 0.71 \\
\hline Age $<40$ years & 40.3 & $-22.4(4.2)$ & 40.3 & $-13.5(6)$ & 184 & $35.3(4.9)$ & 9.2 & $10.7(1.7)$ & 4.5 & $8(1.9)$ \\
\hline Age $\geq 40$ years & 38.7 & $-15.3(9.5)$ & 39.7 & $-23.6(4)$ & 220 & 24.1(7) & 9.5 & $8.1(1.9)$ & 4.5 & $8.7(0.9)$ \\
\hline Pvalue & 0.65 & 0.54 & 0.90 & 0.19 & 0.006 & 0.18 & 0.27 & 0.31 & 0.96 & 0.75 \\
\hline
\end{tabular}

N.B: BMI, body mass index; HAART, highly active antiretroviral therapy; Hb, hemoglobin; PLT, platelets; WBC, white blood cells; CD4+, clusters of differentiation; AST, Aspartate aminotransferase; ALT, alanine aminotransferase; ALP, Alkaline phosphatase; TP, Total protein; ALB, Albumin; ; NNRTI, nonnucleoside reverse transcriptase inhibitors; EFV, Efavirenz; NVP, Nevirapine; NRTI, nucleoside reverse transcriptase inhibitors; AZT, Zidovudine; TDF, Tenofovir ; *mean, mean at switch 0 month

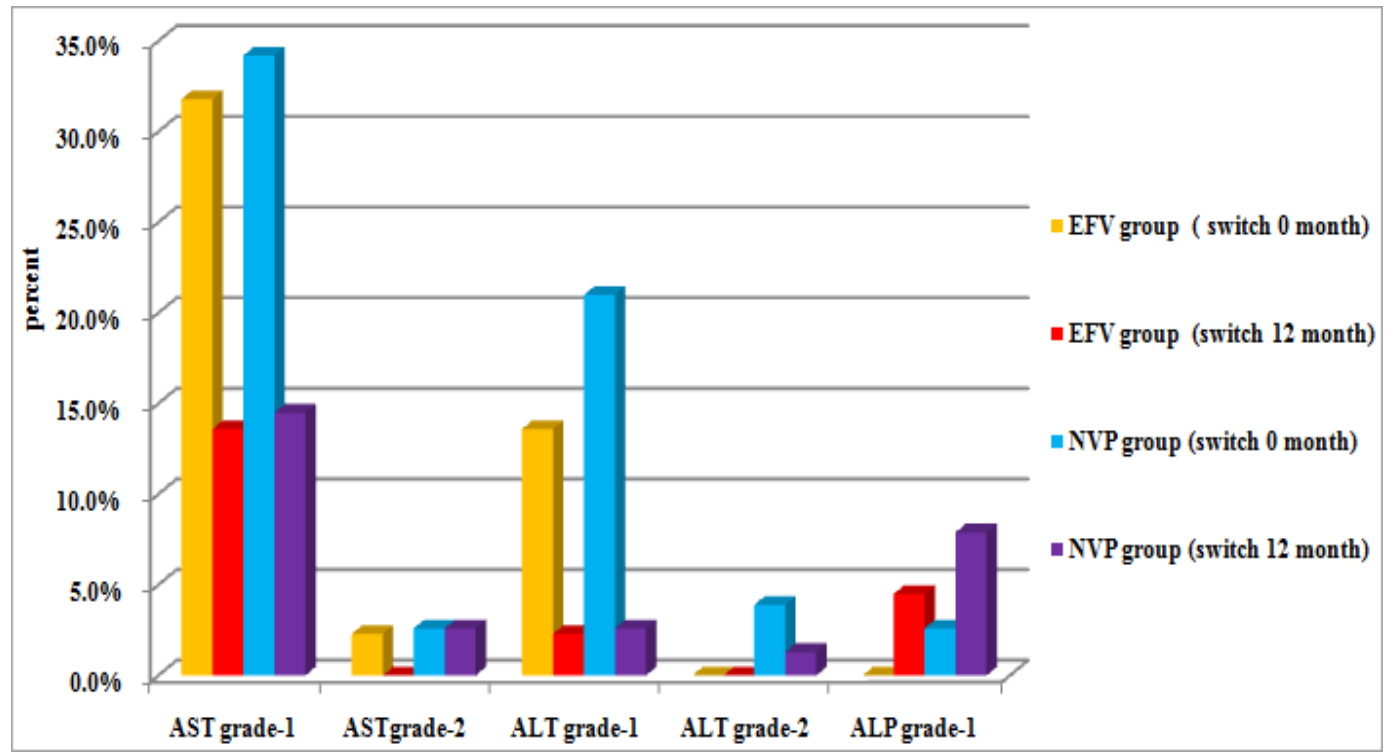

Figure 2. Comparison of hepatotoxicity indicator serum liver enzymes grade of the switch group with use of NNRTIs 


\section{Discussion}

Following recommendations from WHO 2010 guidelines, in $2013 \mathrm{~d} 4 \mathrm{~T}$ was phased-out in Ethiopia from adults' treatment [11].The replacing of d4T + lamivudine (3TC) with Zidovudine $(\mathrm{AZT})+3 \mathrm{TC}$ is not as a result of immunological failure but for the risks of metabolic and anthropometric alterations in HIV-infected patients. Therefore as expected, effectiveness of immunologic competence remained steady after therapy change. The present study result shows insignificant difference in CD4 cell count of the switch group when compared to control group at the 12 month of therapy change and which was similar with a study conducted in Germany [12].

Our study indicates that serum mean AST and ALT level in the switch group decreased significantly over the time observed ( $\mathrm{p}=<0.0001$ for both enzyme); the mean ALP level was significantly increased $(\mathrm{p}=<0.0001)$. And hepatotoxicity in the switch group was significantly decreased (ALT grade $\geq$ 1 was $3.3 \%$ ) after one year of post switch. This finding is in line with the reports of incidence of drug-related hepatitis in US and European trials (ranged from 1\% to 10\%) [13-16].

We found that the proportions of hepatotoxicity after 12 month of post switch, which was higher among patients using NVP-based regimen when compared to EFV but not significant (ALT $\geq$ grade-1 was $3.9 \%$ vs. $2.3 \%$ ). Ogedegbe $e t$ $a l$. have reported that all classes of antiretrovirals have been associated with increases in serum transaminases, the nonnucleoside reverse transcriptase inhibitors (NNRTIs) and protease inhibitors (PIs) appear most commonly associated with inducing liver enzyme, [17]. Our finding is comparable with cohort studies report from Haiti, Thailand, India, Zambia, and Malawi: which was ranging from $<1 \%$ to $7 \%$ for NVP-associated hepatotoxicity [18,19-23]. Also in similar with our finding, studies have shown higher frequencies of hepatotoxicity in patients using NVP-based regimen (4-18\%) when compared to those using EFV (1-8\%) $[6,9,24-26]$.

A study from South African reported that $17 \%$ incidence of serious hepatotoxicity (i.e., ALT and AST levels 5times upper limit normal $(5 x U L N))$ [27]. Contrary to this report, our cohort indicates no patients were seen with AST and ALT level greater than 2xULN.

Our study indicates patients switched from d4T/3TC to AZT/3TC had drastically reduced hepatotoxicity when compared with switch baseline. Moreover, hepatotoxicity due to hepatic steatosis resulting from mitochondrial toxicity was more common with d4T than other NRTIs [9] and Ogedegbe et al. report also reflected similar suggestion [17].

The mean AST and ALT were significantly higher in males when compared to females at the switch baseline but no significant hepatotoxicity was observed in patients BMI. On the contrary to our findings, one study revealed that female patients with a BMI $<18.5 \mathrm{Kg} / \mathrm{m}^{2}$ had a $50 \%$ incidence of serious hepatotoxicity [28].

\section{Conclusion}

In summary, the proportions of abnormally raised liver enzymes (AST and ALT) were significantly decreased at 12 months of post switch. These significant improvements in hepatotoxicity indicator serum liver enzymes suggest that a substitution of d4T to AZT may reverse hepatotoxicity in HIVtreated patients whereas immunologic change remained stable.

However a significant decrease in WBC count after therapy replacement should be requiring strict observation; and a significantly raised ALP enzyme should be monitored periodically to observe the progress and also have a need of further investigations: like bone function tests and 5'nucleotidase enzyme test to identify the source of this enzyme.

\section{Competing Interests}

We announce that we have no any competing interests.

\section{Authors' Contributions}

D. Assegu generated and designed the study, A. Tadewos performed analysis and interpretation of data including with manuscript drafting and the critical appraisal.

\section{Acknowledgements}

We want to acknowledge the laboratory technologists and technicians of the Hawassa University teaching hospital and ART clinic staffs for their immeasurable support during data collection. Our gratefulness is also extended to Hawassa University referral Hospital for great support; and HIV/AIDS patients for their eagerly involvement in the study.

\section{References}

[1] F.J. Palella, K.M. Delaney, A.C. Moorman, M.O. Loveless, J. Palella FJ, Delaney KM, Moorman AC, Loveless MO, Fuhrer J, Satten GA, et al. Declining morbidity and mortality among patients with advanced human immunodeficiency virus infection. N Engl J Med 1998; 338(13): 853-60.

[2] Kalyesubula R, Kagimu M, Opio KC, Kiguba R, Semitala CF, Schlech WF et al. Hepatotoxicity from first line antiretroviral therapy: an experience from a resource limited setting. Afr Health Sc 2011; 11(1): 16 -23.

[3] WHO. Anti-retroviral therapy for HIV infection in adults and adolescents 2006.

http://www.who.int/hiv/pub/arv/adult2010/en/index.html. accessed on 02 Oct 2014

[4] Mankhatithan W, Lueangniyomkul A, Manosuthin W. Hepatotoxicity in patients co-infected coinfected with tuberculosis and HIV-1 while receiving non-nucleoside reverse transcriptase inhibitors based antiretroviral therapy and rifampicin containing antituberculosis drugs. Southeast Asian J Trop Med Public Health 2011; 42(3): 651-58. 
[5] Sulkowski MS. Drug-induced liver injury associated with antiretroviral therapy that includes HIV-1 protease inhibitors. Clin Infect Dis 2004; 38(2):90-7.

[6] Rivero A, Mira JA, Pineda JA. Liver toxicity induced by nonnucleoside reverse transcriptase inhibitors. J Antimicrobial Chemotherapy 2007; 59(3): 342-6.

[7] Brück S, Witte S, Brust J, Schuster D, Mosthaf F, Procaccianti $\mathrm{M}$ et al. Hepatotoxicity in patients prescribed Efavirenz or Nevirapine. Euro J Med Res 2008; 13(7): 343-48.

[8] WHO. Consolidated Guidelines on the Use of Antiretroviral Drugs for Treating and Preventing HIV Infection: Recommendations for a Public Health Approach.. http://www.who.int/about/licensing/copyright_form/en/index. html. accessed on 02 Oct 2014.

[9] Gallant JE, Hoffmann CJ. Johns Hopkins HIV guideline of Hepatotoxicity. http://www.hopkinsguides.com/hopkins/ub/view/Johns_Hopki ns_HIV_Guide/545091/all//Hepatotoxicity. accessed on 05 Oct 2014

[10] Leth F, Phanuphak P, Stroes E, Gazzard B, Cahn P, Raffi FRP et al. Nevirapine and efavirenz elicit different changes in lipid profiles in antiretroviral therapy-naïve patients infected with HIV-1. PLoS Med 2004; 1(1): e19.

[11] WHO. Antiretroviral Therapy for HIV Infection in Adults and Adolescents: Recommendations for a Public Health Approach. World Health organization, 2010. http:// whqlibdoc.who.int/publications/2010/9789241599764_eng.pd f. accessed on 03 Oct 2014

[12] Claas GJ, Jülg B, Röling J, Goebel FD, Bogner JR. Metabolic and anthropometric changes one year after switching from didanosine/stavudine to tenofovir in HIV-infected patients. Eur J Med Res 2007; 12: 54-6.

[13] Pollard R, Robinson P, Dransfield K. Safety profile of nevirapine, a non-nucleoside reverse transcriptase inhibitor for the treatment of human immunodeficiency virus infection. Clin Ther 1998; 20(6):1071-92.

[14] Martinez E, Blanco J, Arnaiz J. Hepatotoxicity in HIV-1infected patients receiving nevirapine-containing antiretroviral therapy. AIDS 2001; 15(10): 1261-8.

[15] Stern J, Robinson P, Love J, Lanes S, Imperiale M, Mayers D. A comprehensive hepatic safety analysis of nevirapine in different populations of HIV infected patients. J Acquir Immune Defic Syndr 2003;. 34 (Suppl 1): 21-33.

[16] Wit F, Weverling G, Jurriaans S, Lange J. Incidence of and risk factors for severe hepatotoxicity associated with antiretroviral combination therapy. J Infect Dis 2002; 186: 23 31 .

[17] Ogedegbe AO, Sulkowski MS. Antiretroviral-associated liver injury. Clin Liver Dis 2003; 7(2): 475-99.
[18] Severe P, Leger P, Charles M, Noel F, Bonhomee G, Bios G et al. Antiretroviral therapy in a thousand patients with AIDS in Haiti. N Eng J Med 2005; 353(22):2325-34.

[19] Kumarasamy N, Lai A, Cecelia AJ, Saghayam S, Solomon S, Flanigan $\mathrm{T}$ et al. Toxicities and adverse events following generic HAART in south Indian HIV-infected individuals: In proceedings of the 7th International Congress on Drug Therapy and HIV Infection. Glasgow, United Kingdom, 2004.

[20] Anekthananon T, Ratanasuwan W, Techasathit W, Sonjai A, Suwanagool S. Safety and efficacy of a simplified fixed-dose combination of stavudine, lamivudine and nevirapine (GPOVIR) for the treatment of advanced HIV-infected patients: a 24-week study. J Med Assoc Thai 2004; 87(7): 760-7.

[21] Pujari SN, Patel AK, Naik E, Patel KK, Dravid A, Patel JK et al. Effectiveness of generic fixed-dose combinations of highly active antiretroviral therapy for treatment of HIV infection in India. J Acquir Immune Defic Syndr 2004; 37(5):1566-9.

[22] Oosterhout JJ, Bodasing N, Kumwenda JJ, Nyirenda C, Mallewa J, Cleary PR et al. Evaluation of antiretroviral therapy results in a resource-poor setting in Blantyre, Malawi. Trop Med Int Health 2005; 10(5): 464-70.

[23] Cantrell R, Chi B, Mulenga L, Sinkala M, Levy J, Stringer E et al. Incidence and predictors of hepatotoxicity among patients receiving nevirapine (NVP)-containing antiretroviral therapy (ART) in Zambia: in Program and abstracts of the 16th International AIDS Conference (Toronto, Canada). Toronto, Canada: International AIDS Society, 2006.

[24] Reisler R, Liou S, Servoss J, Robbins G, Theodore D, Murphy $\mathrm{R}$ et al. Incidence of hepatotoxicity and mortality in 21 adult antiretroviral treatment trials: ACTG Liver Diseases Focus Group. 1st International AIDS Society Conference on HIV Pathogenesis and Treatment, 2001.

[25] Manfredi R, Calza L, Chiodo F. Efavirenz versus Nevirapine in current clinical practice: A prospective, open-label observational study. JAIDS, 2004; 35(5):492-502.

[26] Leth F, Phanuphak P, Ruxrungtham K, Baraldi E, Miller S, Gazzard B et al. Comparison of first-line antiretroviral therapy with regimens including Nevirapine, efavirenz, or both drugs, plus stavudine and lamivudine: a randomized open-label trial, the 2NN study. Lancet 2004; 363(9417):1253-63.

[27] Sanne I, Mommeja-Marin H, Hinkle J, Bartlett JA, Lederman MM, Maartens $\mathrm{G}$ et al. Severe hepatotoxicity associated with nevirapine use in HIV-infected subjects. J Infect Dis 2005; 191 (6): 825-829.

[28] Leith L, PilieroP, Storfer S, Mayers D, Hinzmann R. Appropriate use of nevirapine for long- term therapy. J Infect Dis 2005; 192(3):545-6, 2005. 